ALEA, Lat. Am. J. Probab. Math. Stat. 14, 249-270 (2017)

DOI: 10.30757/ALEA.v14-15

\title{
Directed Diffusion-Limited Aggregation
}

\section{Sébastien Martineau}

Faculty of Mathematics and Computer Science

The Weizmann Institute of Science

234 Herzl Street

Rehovot 7610001 Israel

E-mail address: sebastien.martineau@weizmann.ac.il

URL: http://perso.ens-lyon.fr/sebastien.martineau

\begin{abstract}
In this paper, we define a directed version of the Diffusion-LimitedAggregation model. We present several equivalent definitions in finite volume and a definition in infinite volume. We obtain bounds on the speed of propagation of information in infinite volume and explore the geometry of the infinite cluster. We also explain how these results fit in a strategy for proving a shape theorem for this model.
\end{abstract}

\section{Introduction}

Diffusion-Limited Aggregation (in short, DLA) is a statistical mechanics growth model that has been introduced by Witten and Sander (1981). It is defined as follows. A first particle - a site of $\mathbb{Z}^{2}$ - is fixed. Then, a particle is released "at infinity" and performs a symmetric random walk. As soon as it touches the first particle, it stops and sticks to it. Then, we release another particle, which will also stick to the cluster (the set of the particles of the aggregate), and so on... After a large number of iterations, one obtains a fractal-looking cluster.

DLA does not just model sticking particles, but also Hele-Shaw flow (see Shraiman and Bensimon, 1984), dendritic growth (see Vicsek, 1992) and dielectric breakdown (see Brady and Ball, 1984). Figure 1.2 illustrates the viscous fingering phenomenon, which appears in Hele-Shaw flow. This phenomenon can be observed by injecting quickly a large quantity of oil into water.

This model is extremely hard to study; only two non-trivial results are rigorously known about DLA: an upper bound on the speed of growth (see Kesten, 1987) and the fact that the infinite cluster has almost surely infinitely many holes, i.e. that

Received by the editors December 16th, 2015; accepted March 08th, 2017.

2010 Mathematics Subject Classification. 82C24 (and 82C22, 82C41, 82C43, 60K35).

Key words and phrases. Diffusion-Limited Aggregation, Ballistic deposition. 


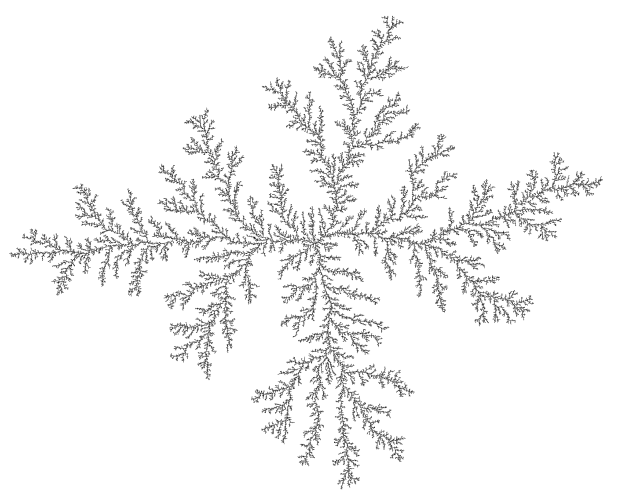

Figure 1.1. DLA cluster obtained by Vincent Beffara.

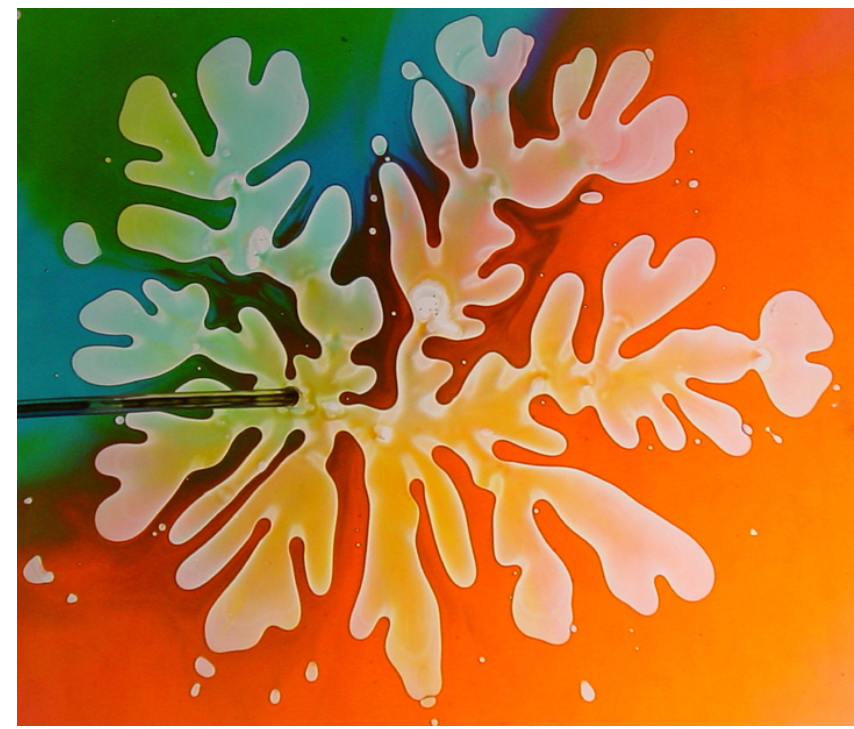

FiguRE 1.2. Viscous fingering picture obtained by Jessica Todd.

the complement of the cluster has infinitely many finite components (see EberzWagner, 1999). The difficulty comes from the fact that the dynamics is neither monotone nor local, and that it roughens the cluster.

The non-locality is quite clear: if big arms surround $P$, even if they are far from it, $P$ will never be added to the cluster.

By non-monotonicity (which is a more serious issue), we mean that there is no coupling between a DLA starting from a cluster $C$ and another from a cluster $D \subsetneq C$ such that, at each step, the inclusion of the clusters remains valid almost 
surely. To understand why, throw the same particles for both dynamics, i.e. use the naïve coupling. The big cluster will catch the particles sooner than the small one: when a particle is stopped in the $C$-dynamics ${ }^{1}$, it may go on moving for the $D$-dynamics and stick somewhere that is not in the $C$-cluster, which would break the monotonicity. In fact, this is even a proof of the non-existence of any monotonic coupling, under the assumption that there exists $(P, Q) \in D \times(C \backslash D)$ such that if $R \in\{P, Q\}, R$ can be connected to infinity by a $\mathbb{Z}^{2}$-path avoiding $C \backslash\{R\}$.

Finally, the fact that the dynamics roughens the cluster instead of smoothing it is what makes the difference between the usual (external) DLA and the internal DLA of Diaconis and Fulton (1991), for which a shape theorem exists (see Lawler et al., 1992). Even though this roughening is not mathematically established, simulations such as the one of Figure 1.1 suggest it by the fractal nature of the picture they provide.

The rigorous study of DLA seeming, for the moment, out of reach, several toy models have been studied. These models are usually easier to study for one of the following reasons:

- either the particles are not added according to the harmonic measure of the cluster (i.e. launched at infinity) but "according to some nicer measure" ${ }^{2}$;

- or the dynamics does not occur in the plane ${ }^{3}$.

In this paper, we prove some results on Directed Diffusion-Limited Aggregation (DDLA), which is a variant where the particles follow downward directed random walks. A large cluster is presented in Figure 1.3. Directed versions of DLA have already been considered by physicists ${ }^{4}$ but, to our knowledge, they have been rigorously studied only in the case of the binary tree (or Bethe lattice). The present model is defined in the plane. Simulations strongly suggest that the DDLA-cluster

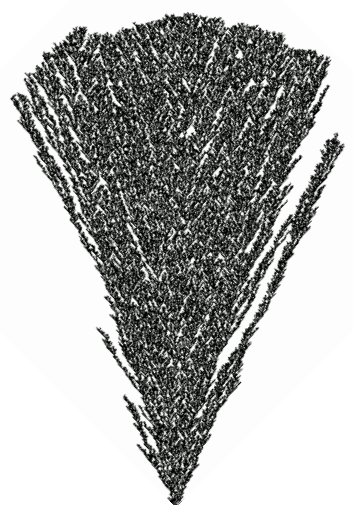

FiguRE 1.3. Large DDLA cluster obtained by Vincent Beffara.

\footnotetext{
$1_{\text {and }}$ if, at the considered time, the $C$-cluster is still bigger than the $D$-one. .

${ }^{2}$ See e.g. Carleson and Makarov (2002).

${ }^{3}$ See e.g. Benjamini and Yadin (2008) for a study of DLA on cylinders $G \times \mathbb{N}$ or Amir et al. (2016, 2013); Amir (2017+) for results on long-range DLA on $\mathbb{Z}$.

${ }^{4}$ See Bradley and Strenski (1984, 1985); Majumdar (2003).
} 
converges after suitable rescaling to some deterministic convex compact, delimited from below by two segments.

DDLA can be seen either as a ballistic deposition model where the falling particles fluctuate randomly or as a stretch version of DLA. See respectively Seppäläinen (2000) and Berger et al. (2014). See also Johansson Viklund et al. (2012) for a study of the Hastings-Levitov version of DDLA; Johansson Viklund et al. (2012) and the present paper have been written independently.

Let us now review the main results of this paper. In Section 2, we define DDLA in discrete and continuous time. We also provide equivalent definitions in terms of downward directed random walks that fall onto the aggregate and stick to it, and in terms of upward directed random walks released from the aggregate and trying to avoid it. The upward version in continuous time is suitable for defining DDLA in infinite volume. This is performed in Section 3. We argue in Section 2 why we believe that defining this infinite volume version of DDLA should be an ingredient in the computation of the conjectured asymptotic shape (see Conjecture 2.3). We get the following upper bound on how far we need to go to define the dynamics on a finite set during a finite time.

Notation. Throughout the paper, we use "a.s.e." as an abbreviation for "almost surely, eventually", which means either "almost surely, there exists $n_{0} \in \mathbb{Z}_{+}$such that for all $n \geq n_{0}$ " or "almost surely, there exists $t_{0} \in \mathbb{R}_{+}$such that for all $t \geq t_{0}$ ".

Theorem 3.4. Let $F$ be a non-empty finite subset of $\mathbb{Z}^{2}$. Consider continuous time DDLA with the following initial configuration: outside $F$, a vertex is in the aggregate at time 0 if and only if its height is 0; at every point of $F$, it is unknown whether it is initially empty or occupied. If we denote by $\mathfrak{h}_{t}$ the height of the set of vertices with unknown status at time $t$ and by $\mathfrak{d}_{t}$ its (horizontal) width, then a.s.e.

$$
\mathfrak{h}_{t} \leq c_{0} \cdot t \ln t \text { and } \mathfrak{d}_{t} \leq c_{0} \cdot t^{2} \ln t
$$

for some deterministic constant $c_{0}$ independent of $F$.

In Section 4, we use Kesten's argument (Kesten, 1987) to obtain bounds on the speed of horizontal growth and vertical growth of the cluster. We get the following result.

Theorem 4.1. Consider discrete time DDLA initiated with $\{(0,0)\}$. Denote by $\mathfrak{h}_{n}$ the height of the cluster at time $n$, and by $\mathfrak{d}_{n}$ its width. For some constant $c_{1}$, almost surely, eventually,

$$
\begin{gathered}
\sqrt{2 n} \leq \mathfrak{h}_{n} \leq c_{1} n^{2 / 3} \\
\text { and } \\
c_{1}^{-1} n^{1 / 3} \leq \mathfrak{d}_{n} \leq c_{1} \sqrt{n} .
\end{gathered}
$$

As far as Kesten's argument is concerned, it is in Lemma 4.4 that the difference between DLA and directed DLA becomes apparent.

Finally, Section 5 explores the geometry of the infinite cluster. In a very weak sense, "Corollary 5.3 states that the cluster does not grow sideways as much as we could have imagined, and Proposition 5.4 roughly indicates that the cluster tends to grow in a vertical fashion".

Corollary 5.3. Launch a DDLA from $\{(0,0)\}$. Almost surely, for every $n \in \mathbb{Z}_{+}$, only finitely many points of $\left(\mathbb{Z}_{+} \times\{n\}\right) \cup\left(\{n\} \times \mathbb{Z}_{+}\right)$are added to the cluster. 
Proposition 5.4. Consider a DDLA starting from $C \neq \varnothing$. Let

$$
C_{\infty}:=\bigcup_{t \geq 0} C_{t} .
$$

Then, almost surely, every open cone which contains a vertical half-line intersects $C_{\infty}$ at infinitely many points.

Section 2 is used in subsequent sections. Apart from that, all sections are independent, and may thus be read in any order. They are all conceived as very humble steps towards a shape theorem: each of them brings a very weak answer to an important question. Section 3 indicates that the dependencies in the model are not completely wild. Section 4 estimates the horizontal and vertical speed of growth of the model: understanding the scaling is the first step in proving a shape theorem. At last, the conjectured asymptotic shape is delineated below by two segments, and simulations suggest that this angle is strictly less than $\frac{\pi}{2}$. In Section 5 , we prove a very weak form of this.

\section{Presentation of DDLA}

2.1. Some notation. In this paper, when dealing with DDLA, we will think of $\mathbb{Z}^{2}$ as rotated by an angle of $+\frac{\pi}{4}$ (so that the particles we will throw move downward). The vertices of $\mathbb{Z}^{2}$ will often be referred to as sites. Let

$$
\mathbf{E}:=\left\{((a, b),(c, d)) \in\left(\mathbb{Z}^{2}\right)^{2}:(a=c \& b=d+1) \text { or }(a=c+1 \& b=d)\right\}
$$

be the set of the (directed) edges; it endows $\mathbb{Z}^{2}$ with a structure of directed graph. We will denote by $d$ the graph-distance on $\left(\mathbb{Z}^{2}, \mathbf{E}\right)$, i.e. the $\|\cdot\|_{1}$-distance. If $e=(P, Q)$ is an edge, we call $P$ the upper vertex of $e$ and $Q$ its lower vertex. They are referred to as $\mathbf{u}(e)$ and $\mathbf{l}(e)$.

A downward directed symmetric random walk is a Markov chain with transition probabilities

$$
p(P, Q)=\mathbf{1}_{(P, Q) \in \mathbf{E}} / 2 .
$$

An upward directed symmetric random walk is obtained with transition probabilities

$$
p(P, Q)=\mathbf{1}_{(Q, P) \in \mathbf{E}} / 2 .
$$

When the starting point of a directed random walk is not specified, it is tacitly taken to be $(0,0)$.

The height of $P=(a, b)$, denoted by $\mathbf{h}(P)$, is $a+b$. Its horizontal deviation (relative to 0 ) is $\mathbf{d}(P):=b-a$. The height (resp. horizontal deviation) of $P$ relative to $Q$ is $\mathbf{h}(P)-\mathbf{h}(Q)$ (resp. $\mathbf{d}(P)-\mathbf{d}(Q)$ ). If $A \subset \mathbb{Z}^{2}$, we set

$$
\mathbf{h}(A):=\sup _{P \in A} \mathbf{h}(P), \mathbf{d}(A):=\sup _{P \in A} \mathbf{d}(P) \text { and }|\mathbf{d}|(A):=\sup _{P \in A}|\mathbf{d}(P)| .
$$

The line of height $n$ is

$$
L_{n}:=\left\{(x, y) \in \mathbb{Z}_{+}^{2}: x+y=n\right\} .
$$

We also set

$$
L_{\leq n}:=\left\{(x, y) \in \mathbb{Z}_{+}^{2}: x+y \leq n\right\} .
$$

A line $L_{n}$ is said to be above a set $S$ if $S \subset L_{\leq n-1}$. Finally, if one fixes a subset $C$ of $\mathbb{Z}^{2}$, the activity of a site $P \in \mathbb{Z}^{2}$ relative to $C$ is

$$
\operatorname{act}_{C}(P):=\mathbb{P}\left[\forall n \in \mathbb{Z}_{+}, P+W_{n} \notin C\right] \cdot|\{e \in \mathbf{E}: \mathbf{l}(e) \in C \& \mathbf{u}(e)=P\}|,
$$


where $\left(W_{n}\right)_{n \in \mathbb{Z}_{+}}$is an upward directed symmetric random walk and $\mid$. $\mid$stands for the cardinality operator. In what follows, we will consider a growing subset of $\mathbb{Z}^{2}$, called cluster. The current activity (or activity) of a site $P$ will then be relative to the cluster at the considered time. The activity of the cluster will be the sum over all sites of their activity.

2.2. Definition in discrete time. At time 0, the cluster is $C_{0}:=\{(0,0)\}$. Assume that the cluster has been built up to time $n$, and that $C_{n} \subset L_{\leq n}$. To build $C_{n+1}$, choose any line $L_{k}$ above $C_{n}$. Then, independently of all the choices made so far, choose uniformly a point in $L_{k}$, and send a downward symmetric random walk $\left(W_{n}\right)$ from this point. If the walk intersects $C_{n}$, then there must be a first time $\tau$ when the walker is on a point of the cluster: let

$$
C_{n+1}:=C_{n} \cup\left\{W_{\tau-1}\right\} \subset L_{\leq n+1} .
$$

If the random walk fails to hit the cluster, we iterate the procedure \{choice of a starting point + launching of a random walk $\}$ independently and with the same $k$, until a random walk hits the cluster, which will happen almost surely. This is obviously the same as conditioning the procedure to succeed.

The dynamics does not depend on the choices of $k$ : indeed, choosing uniformly a point in $L_{k+1}$ and taking a step downward give the same measure to all the points of $L_{k}$ (and if a walker goes outside $\mathbb{Z}_{+}^{2}$, it will never hit the cluster). The dynamics is thus well-defined. We call this process Directed Diffusion-Limited Aggregation (or DDLA).

Remark 2.1. Since the process does not depend on the choices of $k$, we can take it as large as we want so that we may (informally at least) think of the particles as falling from infinity.

Here is another process, which is the same (in distribution) as DDLA. We set $C_{0}:=\{(0,0)\}$. Assume that we have built $C_{n}$, a random set of cardinality $n+1$. We condition the following procedure to succeed:

Procedure. We choose, uniformly and independently of all the choices made so far, an edge $e$ such that $\mathbf{l}(e) \in C_{n}$. We launch an upward directed symmetric random walk from $\mathbf{u}(e)$. We say that the procedure succeeds if the random walk does not touch $C_{n}$.

The particle added to the cluster is the upper vertex of the edge that has been chosen. Iterating the process, we obtain a well-defined dynamics. It is the same as the first dynamics: this is easily proved by matching downward paths with the corresponding upward ones.

2.3. Definition in continuous time. We now define DDLA in continuous time: this is the natural continuous time version of the second definition of DDLA. Let $\left(\left(N_{t}^{e}\right)_{t \geq 0}\right)_{e \in \mathbf{E}}$ be a family of independent Poisson processes of intensity 1 indexed by the set of the directed edges. The cluster $C(0)$ is defined as $\{(0,0)\}$ and we set $T(0):=0$.

Assume that for some (almost surely well-defined) stopping time $T(n)$, the cluster $C(T(n))$ contains exactly $n$ particles. Then, wait for an edge whose lower vertex is in $C(T(n))$ to ring (such edges will be called growth-edges). When the clock on a growth-edge $e$ rings, send an independent upward directed random walk from 
its upper vertex. If it does not intersect $C(T(n))$, add a particle at $\mathbf{u}(e)$ and define $T(n+1)$ to be the current time. Otherwise, wait for another growth-edge to ring, and iterate the procedure.

This dynamics is almost surely well-defined for all times ${ }^{5}$ because it is stochastically dominated by first-passage percolation (Kesten, 1986). Markov chain theory guarantees that $\left(C_{n}\right)_{n \in \mathbb{Z}_{+}}$and $(C(T(n)))_{n \in \mathbb{Z}_{+}}$are identical in distribution.

Remark 2.2. This definition in continuous time consists in adding sites at a rate equal to their current activity.

2.4. Some general heuristics. Before going any further, it may be useful to know what is the theorem we are looking for and how the results presented in this paper may play a part in its proof. In this subsection, we present highly informal heuristics that have not been made mathematically rigorous in any way yet. They constitute a strategy for proving a shape theorem for DDLA.

Conjecture 2.3. There is some convex compact $D$ of non-empty interior such that $\frac{C(t)}{t}$ converges almost surely to $D$ for the Hausdorff metric. Furthermore, the boundary of $D$ consists in two segments and the $(-\pi / 4)$-rotated graph of a concave function.

To prove such a result, the step 0 may be to prove that the width and height of the cluster both grow linearly in time, so that we would know that we use the right scaling. This would result from a stronger form of Proposition 4.8.

Provided this, one may use compactness arguments to prove that if there exists a unique "invariant non-empty compact set" $D$, then we have the desired convergence (to $D$ ). By invariance, we informally mean the following: if $t$ is large enough and if we launch a DDLA at time $t$ from $(t D) \cap \mathbb{Z}^{2}$, then $\frac{C(t+s)}{t+s}$ "remains close" to $D$.

This existence and uniqueness may be proved by finding a (maybe non-explicit) ordinary differential equation satisfied by the upper interface of $D$. To do so, we would proceed in two steps.

Step 1. First of all, one needs to check that the upper interface is typically "more or less" the $(-\pi / 4)$-rotated graph of a differentiable function. To do so, one would need to control fjords. Roughly speaking, we call fjord the area delimited by two long and close arms of the cluster. Fjords are the enemies of both regularity and "being the graph of a function".

Here are some heuristics about fjords: in Figure 1.3, we observe that there are mesoscopic fjords far from the vertical axis and no such fjord close to it. We try to account for this.

Definition 2.4. We say that a site $P$ shades a second one if it can catch particles that would go to the second site if $P$ was vacant.

Assume that we have a behaviour as suggested by Figure 1.3. If we are close to the vertical axis, the local slope is close to 0 . We will assume that, at any time, none of the two top-points of the arms delineating the considered fjord shades the other: they will thus survive (i.e. keep moving), following more or less upward directed random walks. By recurrence of the 2-dimensional random walk, we obtain that the two top-points will collide at some time, closing the fjord. To avoid the shading

\footnotetext{
$5_{\text {i.e. }} \sup _{n} T(n)$ is almost surely infinite
} 
phenomenon, one needs a still unknown proper and tractable definition of top-point. However, it seems quite reasonable to expect this phenomenon "not to occur" if the slope is close to 0 because there is no initial shading.

When the slope gets higher, the shading phenomenon appears. If the slope is not too high, the "lower top-point" manages to survive but it is hard for it to catch up with the upper one: this creates a fjord 6 . If the slope is too high, the "lower top-point" stops catching particles: we are in the lower interface.

Step 2. Now, we need to find an ODE satisfied by $r$, where $\alpha \mapsto r(\alpha)$ is the angular parametrization of the upper interface of $D$ and is defined on $\left(-\alpha_{0}, \alpha_{0}\right)$. We assume that $\alpha=0$ corresponds to what we think of as the vertical.

Assume that one can launch a DDLA from an infinite line of slope $\tan (\theta)-$ which is made possible by Section 3 when $\tan (\theta) \in(-1,1)$, and can actually be done in general - and define a deterministic ${ }^{7}$ speed of vertical growth $\mathbf{v}(\alpha)$. The set $D$ being invariant, $r(\alpha) \cdot \cos (\alpha)$ must be proportional to $\mathbf{v}(\theta(\alpha))$, where $\tan (\theta(\alpha))$ stands for the local slope of $D$ at the neighborhood of the point defined by $\alpha$ and $r(\alpha)$.

More exactly, we have

$$
\left\{\begin{array}{c}
r(\alpha) \cdot \cos (\alpha)=c \cdot \mathbf{v}(\theta(\alpha)) \\
\tan (\alpha-\theta(\alpha))=\frac{r^{\prime}(\alpha)}{r(\alpha)} .
\end{array}\right.
$$

The knowledge of $\theta\left(\alpha_{0}\right)$ due to the previous step allows us to find $\alpha_{0}$.

Simulations suggest that $\alpha_{0}<\pi / 4$; Corollary 5.3 is a weak result in this direction.

The last point that has to be checked is that the lower interface consists of two segments. Assume that the points of the lower interface are of bounded local slope. From this and large deviation theory, one can deduce that it costs typically exponentially much for a particle to stick to the lower interface at large distance from the upper interface. ${ }^{8}$ This might allow us to compare DDLA with ballistic deposition, for which the upper interface converges to the graph of a concave function (see Seppäläinen, 2000) and the lower interface converges to the union of two segments (use the Kesten-Hammersley Lemma, see Smythe and Wierman, 1978).

\section{Bounds on the transport of information}

In this section, we define Directed Diffusion-Limited Aggregation starting from a suitable infinite set. Notice that we make the trivial adjustment that the process now lives in $\mathbb{Z}^{2}$ instead of $\mathbb{Z}_{+}^{2}$.

Here is a very informal description of the construction. Each edge has a Poisson clock and infinitely many upward directed symmetric random walks attached to it, everything being chosen independently. When a clock rings at some edge for the $k^{t h}$ time, if its upper extremity is vacant and its lower one occupied, the $k^{t h}$ random

\footnotetext{
${ }^{6}$ Simulations suggest that this process builds fjords forming a deterministic angle with the vertical.

${ }^{7}$ by ergodicity arguments

${ }^{8}$ By this, we mean that, conditionally on an initial cluster, the probability that the next particle sticks to the lower interface at distance $d$ from the upper interface is lower than $e^{-\epsilon d}$, for some constant $\epsilon$.
} 
walk is sent and we see if it hits the current cluster or not: we add a particle if and only if the walk does not hit the cluster.

In finite volume, this is not problematic because we can (almost surely) define the first (or next) ringing time: since we only need to know the state of the cluster just before we send the walk, the construction is done. In the case of an infinite initial cluster, in any non-trivial time interval, there are almost surely infinitely many ringing times to consider. ${ }^{9}$ To define the dynamics, a solution is to show that, for all $\left(P_{0}, T_{0}\right) \in \mathbb{Z}^{2} \times \mathbb{R}_{+}^{\star}$, what happens at $P_{0}$ before time $T_{0}$ just depends on some random finite set of edges. Indeed, in this case, we can apply the construction in finite volume. This is the idea behind Harris-like constructions. See e.g. Seppäläinen (2000) for an easy Harris-like construction of ballistic deposition, the local and monotonic version of DDLA.

Rigourously, the construction goes as follows. Let $\left(\left(N_{t}^{e}\right)_{t \geq 0}\right)_{e \in \mathbf{E}}$ be a family of independent Poisson processes of intensity 1 indexed by the set of the directed edges. Let $\left(\left(W_{n}^{e, k}\right)_{n \in \mathbb{N}}\right)_{e \in \mathbf{E}, k \in \mathbb{N}^{*}}$ be a family of independent upward directed symmetric random walks (simply referred to as random walks in this section) indexed by $\mathbf{E} \times \mathbb{N}^{\star}$.

Notations. Let $r_{\theta}$ be the rotation of centre $(0,0)$ and angle $\theta$. For $b \in \mathbb{R}_{+}^{\star}$, let

$$
\mathcal{C}_{b}:=r_{-\pi / 4}\left(\left\{(x, y) \in \mathbb{R}^{2}:|y| \geq b|x|\right\}\right)
$$

be the $b$-cone and let

$$
\mathcal{W}_{b}:=r_{-\pi / 4}\left(\left\{(x, y) \in \mathbb{R}^{2}:|y|=(b+1) x\right\}\right)
$$

be the $b$-wedge. (Remember that we think of $\mathbb{Z}^{2}$ as rotated by an angle of $+\pi / 4$.) When $b$ is not specified, it is taken to be equal to the $a$ introduced in the next line.

Assumption on the cluster. There is some $(a, K) \in \mathbb{R}_{+}^{2}$ such that for all $P \in C$,

$$
\left(P+(K, K)+\mathcal{C}_{a}\right) \cap C=\varnothing \text { and }\left(P+s\left((K, K)+\mathcal{C}_{a}\right)\right) \cap C=\varnothing,
$$

where $s$ maps $Q \in \mathbb{Z}^{2}$ to $-Q$.

Let us fix $T_{0} \in \mathbb{R}_{+}^{\star}$. Let us pick a site $P_{0}$ in $\mathbb{Z}^{2}$ and try to decide whether we add it to the cluster before time $T_{0}$ or not and, if so, when. If this can be done with probability 1 , then the dynamics is almost surely well-defined. Indeed, it is enough to check every $\left(P_{0}, T_{0}\right)$ in $\mathbb{Z}^{2} \times \mathbb{Z}_{>0}$.

Definitions 3.1. A site $P$ is said to be activated if there is an upward directed path $\left(Q_{0}, \ldots, Q_{n}\right)$ such that:

- $Q_{0} \in C$,

- $Q_{n}=P$,

- there is an increasing $n$-tuple $\left(t_{1}, \ldots, t_{n}\right)$ such that $t_{n} \leq T_{0}$ and for every $k \in\{1, \ldots, n\}$, the clock at $\left(Q_{k-1}, Q_{k}\right)$ rings at time $t_{k}$.

The model consisting in adding a vertex $P$ before time $t$ if and only if the condition above is satisfied for $t$ instead of $T_{0}$ is called Directed First-Passage Percolation (or DFPP). We also say that a directed edge $(P, Q)$ is activated if there is an upward directed path $\left(Q_{0}, \ldots, Q_{n}\right)$ such that:

\footnotetext{
${ }^{9}$ This problem is essentially the same as the one that makes impossible a discrete-time construction: we cannot throw our particles one after another because there is no uniform probability measure on an infinite countable set.
} 


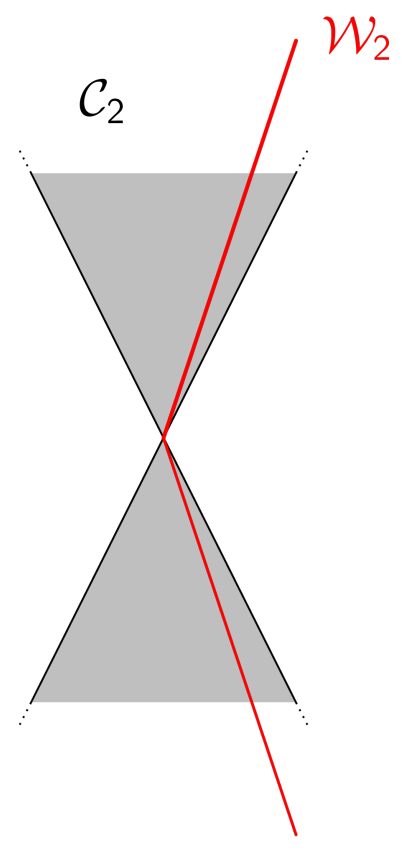

Figure 3.4. The $b$-cone and the $b$-wedge for $b=2$.
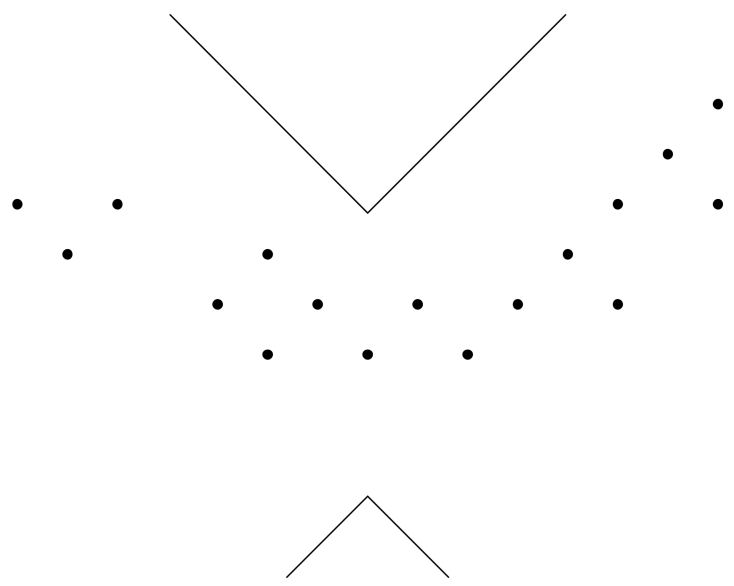

FIGURE 3.5. A finite portion of a cluster satisfying the assumption for $(a, K)=(1,2)$.

- $Q_{0} \in C$,

- $Q_{n-1}=P$ 
- $Q_{n}=Q$,

- there is an increasing $n$-tuple $\left(t_{1}, \ldots, t_{n}\right)$ such that $t_{n} \leq T_{0}$ and for every $k \in\{1, \ldots, n\}$, the clock at $\left(Q_{k-1}, Q_{k}\right)$ rings at time $t_{k}$.

For any directed edge $(P, Q)$, each time the clock at $(P, Q)$ rings, if $(P, Q)$ belongs to the current DFPP cluster, then we launch a new random walk from $Q$; the $k^{\text {th }}$ random walk to be launched is $Q+W^{(P, Q), k}$.

Proposition 3.2. The probability that $P \in \mathbb{Z}^{2}$ is activated decays exponentially fast in $d(P, C)$.

Remark 3.3. Proposition 3.2 is a direct consequence of the exponential decay of subcritical percolation if $T_{0}<\ln 2$.

Proof: Let $B(P, n)$ denote the $\|\cdot\|_{1}$-ball of centre $P$ and radius $n$. Let $k_{0} \in \mathbb{Z}_{>0}$. If the following holds for $n=\left\lfloor\frac{d(P, C)}{k_{0}}\right\rfloor-1$ :

$$
\forall 0<k \leq k_{0}
$$

$B\left(P,\left(k_{0}-k+1\right) n\right)$ contains all the vertices that can be connected to $B\left(P,\left(k_{0}-k\right) n\right)$ by edges whose clock rings between $\frac{k-1}{2}$ and $\frac{k}{2}$,

then $P$ cannot belong to the activation cluster of $C$ for $T_{0} \leq k_{0} / 2$. But, by the exponential decay of activation percolations over a time-range equal to $1 / 2<\ln 2$, the probability that this condition is not satisfied is lower than

$$
\sum_{k=1}^{k_{0}}\left|B\left(P, k_{0} n\right)\right| c e^{-n / c},
$$

which decays exponentially fast in $n$.

It is possible to prove that the dynamics is well-defined in the framework presented above. As this is more technical and less quantitative than what follows, we will not work at that level of generality. We prove bounds on the speed of propagation of the information for a horizontal initial cluster, which imply that one can define the state of a vertex at a given time by looking at finitely many vertices and walks, so that the model is indeed well defined. Such a control guarantees a weak (and quantitative) form of locality, which may help studying further DDLA. Actually, we will see that one can adapt Theorem 3.4 so that it holds in the framework presented above, provided that $a<1$.

Let us consider a DDLA launched with the initial interface

$$
D:=\left\{P \in \mathbb{Z}^{2}: \mathbf{h}(P)=0\right\} .
$$

Before stating the proposition, we need to introduce some terminology. Let $F \Subset \mathbb{Z}^{2}$, i.e. let $F$ be a non-empty finite subset of $\mathbb{Z}^{2}$. We want to define where some information about $F$ may be available. Formally, we want our area of potential influence (a random subset of $\mathbb{Z}^{2}$ depending on time) to satisfy the following property: if we use the same clocks and walks to launch a DDLA from $D$ and one from $D \Delta G$ with $G \subset F$, the clusters will be the same outside the area of potential influence at the considered time. In fact, the way this area is defined in this section, we even know that the pair (area, data of the particles present in the cluster outside the area) satisfies the (say weak) Markov Property. 
We define this area as follows ${ }^{10}$. Instead of saying that a site of $\mathbb{Z}^{2}$ - in the cluster or not - belongs to the area of potential influence, we will say that it is red, which is shorter and more visual. A non-red site belonging to the cluster will be colored in black. Initially,

$$
\mathfrak{R}_{0}:=F
$$

is the red area. Then, a site $P$ becomes red when one of the following events occurs:

- $P=\mathbf{u}(e)$, the site $\mathbf{l}(e)$ is red, the clock on $e$ rings and the launched random walk avoids black sites;

- $P=\mathbf{u}(e)$, the site $\mathbf{l}(e)$ is black, the clock on $e$ rings and the launched random walk avoids black sites and goes through at least one red site.

It is not clear that this is well-defined, for the same reason that makes the definition in infinite volume uneasy, but we will see in the proof of Theorem 3.4 that some larger ${ }^{11}$ set is finite almost surely for all times, so that the construction boils down to finite volume, entailing proper definition of the red area.

By construction, it is clear that if it is well-defined, red is a good notion of area of potential influence.

Notations. $\mathfrak{R}_{t}$ will denote the red area at time $t$. We set $\mathfrak{h}_{t}:=\mathbf{h}\left(\mathfrak{R}_{t}\right)$ and $\mathfrak{d}_{t}:=$ $|\mathbf{d}|\left(\Re_{t}\right)$. This holds only for this section.

Theorem 3.4. If $F \Subset \mathbb{Z}^{2}$ and if we choose $D$ as initial cluster, then $\left(\mathfrak{R}_{t}(F)\right)_{t \geq 0}$ is well-defined and a.s.e.

$$
\mathfrak{h}_{t} \leq c_{0} \cdot t \ln t \text { and } \mathfrak{d}_{t} \leq c_{0} \cdot t^{2} \ln t
$$

for some deterministic constant $c_{0}$ independent of $F$.

Proof: Without loss of generality, we may assume that $\mathfrak{R}_{0}=\{(0,0)\}$. Indeed, if one takes $F$ to be $\{(0,0)\}$, then for any finite subset $G$ of $\mathbf{h}^{-1}\left(\mathbb{Z}_{+}\right)$, the event $G \subset \mathfrak{R}_{1}$ has positive probability.

The rough idea of the proof is the following:

(1) We prove that the red area cannot be extremely wide.

(2) We show that if it is not very wide, it is quite small (in height).

(3) We prove that if it is small, it is narrow.

(4) We initialize the process with the first step and then iterate Steps 2 and Step 3, allowing us to conclude.

Step 1: At most exponential growth. For $n \in \mathbb{Z}_{+}$, we set

$$
S_{n}:=\left\{P \in \mathbb{Z}^{2}: \mathbf{h}(P)+|\mathbf{d}(P)| \leq 2 n \text { and } \mathbf{h}(P)>0\right\} .
$$

We consider the following model.

At time 0 , the cluster is $\mathfrak{S}_{0}:=S_{0}$. An edge $e$ is said to be decisive if $\mathbf{l}(e) \in \mathfrak{S}_{t}$ and $\mathbf{u}(e) \notin \mathfrak{S}_{t}$. The cluster does not change until a clock on a decisive edge rings. When this event occurs, $\mathfrak{S}_{t}$, which was $S_{n}$ for some random $n$, becomes $S_{n+1}$. The data of $\mathfrak{S}_{t}$ is thus just the data of this random $n(t)$.

Let $\left(\tau_{n}\right)_{n \geq 1}$ be a sequence of independent random variables such that $\tau_{n}$ follows an exponential law of parameter $2 n$. Let $T_{n}:=\sum_{k=1}^{n} \tau_{k}$. Then, by construction,

\footnotetext{
${ }^{10}$ Some looser definition may be proposed but this one is used because it is tractable.

${ }^{11}$ Of course, as in all Harris-like constructions, this set is larger than some set that is not defined yet !
} 


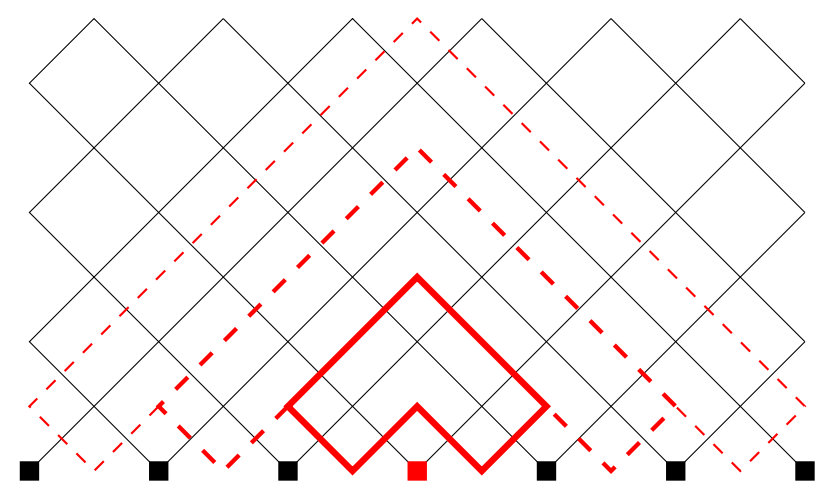

Figure 3.6. Illustration of the model used in Step 1.

$\left(T_{n}\right)_{n \in \mathbb{Z}_{+}}$has the same law as the sequence of the jumping times of the cluster from one state to another.

Proposition 3.5. Almost surely, eventually,

$$
T_{\left\lfloor e^{\left.n^{2}\right\rfloor}\right.}>\frac{n^{2}}{8}
$$

Proof: Consider $f: n \mapsto\left\lfloor e^{n^{2}}\right\rfloor$. By construction, one has the following estimate:

$$
\mathbb{E}\left[\sum_{k=f(n)+1}^{f(n+1)} \tau_{k}\right]=\sum_{k=f(n)+1}^{f(n+1)} \frac{1}{2 k} \underset{n \rightarrow \infty}{\sim} n .
$$

Setting $\mathfrak{T}_{n}:=\sum_{k=f(n)+1}^{f(n+1)} \tau_{k}$, we have

$$
\operatorname{Var}\left[\mathfrak{T}_{n}\right] \underset{\text { indep. }}{=} \sum_{k=f(n)+1}^{f(n+1)} \operatorname{Var}\left[\tau_{k}\right] \leq \frac{1}{4} \times \frac{\pi^{2}}{6}
$$

By Chebyshev's inequality and our control on the expectation, for $n$ large enough,

$$
\mathbb{P}\left[\mathfrak{T}_{n}<\frac{n}{2}\right] \leq \frac{\pi^{2}}{3 n^{2}} .
$$

By the Borel-Cantelli Lemma, a.s.e. $\mathfrak{T}_{n}>\frac{n}{2}$. The result follows.

Consequently, for some (explicit) $c \in \mathbb{R}_{+}^{\star}$, a.s.e. $\mathfrak{S}_{t} \subset S_{\left\lfloor e^{c t}\right\rfloor}$. The area $\mathfrak{R}_{t}$ is therefore well-defined and is a.s.e. a subset of $S_{\left\lfloor e^{c t}\right\rfloor}$.

Step 2: Polynomial growth of the height.

Lemma 3.6. Let $M$ be a sequence of positive real numbers such that a.s.e., $\mathfrak{R}_{n} \subset$ $S_{\left\lfloor M_{n}\right\rfloor}$. Assume that $M_{n}$ is eventually larger than $n$. Then for some constant $a \in \mathbb{R}_{+}^{\star}$, a.s.e., $\mathfrak{h}_{n} \leq$ an $\ln M_{n}$.

Proof: The colored area is the set the sites that are red or black. It is dominated by the directed first-passage percolation starting from $D$ and using the same clocks. 
Let $\mathfrak{P}_{t}$ be the cluster of this percolation at time $t$. We know that, a.s.e. $\mathfrak{R}_{n} \subset$ $S_{\left\lfloor M_{n}\right\rfloor} \cap \mathfrak{P}_{n}=: \mathfrak{A}_{n}^{\exp (c n)}$, where $\mathfrak{A}_{t}^{r}:=S_{\lfloor r\rfloor} \cap \mathfrak{P}_{t}$. For $n \in \mathbb{Z}_{+}$and $a \in \mathbb{R}_{+}^{\star}$,

$$
\begin{aligned}
\mathbb{P}\left[\mathbf{h}\left(\mathfrak{A}_{n}^{M_{n}}\right)>a n \ln M_{n}\right] & \leq \mathbb{P}\left[\exists k \leq 2 n, \mathbf{h}\left(\mathfrak{A}_{\frac{k+1}{2}}^{M_{n}}\right)-\mathbf{h}\left(\mathfrak{A}_{k / 2}^{M_{n}}\right)>a \ln M_{n} / 2\right] \\
& \leq 2 n \max _{k \leq 2 n} \mathbb{P}\left[\mathbf{h}\left(\mathfrak{A}_{\frac{k+1}{2}}^{M_{n}}\right)-\mathbf{h}\left(\mathfrak{A}_{\frac{k}{2}}^{M_{n}}\right)>a \ln M_{n} / 2\right] \\
& \leq 2 n e^{-\operatorname{cst} \cdot a \ln M_{n}}\left(2 M_{n}+1\right) \\
& \leq 2 n\left(2 M_{n}+1\right)^{1-\text { cst } \cdot a} .
\end{aligned}
$$

(For the last inequality, see the last sentence of the proof of Proposition 3.2.) Since $n=$ $O\left(M_{n}\right)$, taking $a$ large enough implies that the probabilities $\mathbb{P}\left[\mathbf{h}\left(\mathfrak{A}_{n}^{M_{n}}\right)>\right.$ an $\left.\ln M_{n}\right]$ are summable. Applying the Borel-Cantelli Lemma, we obtain that a.s.e. $\mathfrak{h}_{n} \leq$ an $\ln M_{n}$.

Applying Lemma 3.6 to $\left(e^{c n}\right)$ and increasing slightly the value of $a$, one gets that a.s.e., $\mathfrak{h}(t) \leq a t^{2}$. Indeed, $(n+1)^{2} \underset{n \rightarrow \infty}{\sim} n^{2}$.

Step 3: Polynomial lateral growth.

Lemma 3.7. Let $M$ be a sequence of real numbers greater than 1 such that a.s.e., $\mathfrak{h}_{n} \leq M_{n}$. Then, for some constant $b \in \mathbb{R}_{+}^{\star}$, a.s.e., $\mathfrak{d}_{n} \leq b \cdot n M_{n}$.

Notation. If $k \in \mathbb{Z}_{+}$, let $H_{k}:=\left\{P \in \mathbb{Z}^{2}: 0 \leq \mathbf{h}(P) \leq k\right\}$ be the $k$-strip.

Proof: Given a natural number $k$, we consider the dynamics defined as in Step 1, but with

$$
S_{n}^{k}:=S_{n} \cap H_{k}
$$

instead of $S_{n}$. We denote by $\mathfrak{S}_{t}^{k}$ the corresponding cluster at time $t$. As long as $\mathfrak{h}_{t} \leq k$, we have $\mathfrak{R}_{t} \subset \mathfrak{S}_{t}^{k}$.

Let $\tau_{n}$ be i.i.d. random variables following an exponential law of parameter 1 and let

$$
T_{n}:=\sum_{i=1}^{n} \tau_{i}
$$

The sequence of the jumping times of the $H_{k}$-dynamics dominates stochastically $\left(T_{n} / 2 k\right)_{n}$.

Large deviation theory guarantees that there is some cst' $^{\prime}$ such that for any $n \in \mathbb{Z}_{+}$,

$$
\begin{aligned}
\mathbb{P}\left[\mathbf{d}\left(\mathfrak{S}_{n}^{M_{n}}\right) \geq\left\lfloor 3 n M_{n}\right\rfloor\right] & \leq \mathbb{P}\left[T_{\left\lfloor 3 n M_{n}\right\rfloor} /\left(2 M_{n}\right) \leq n\right] \\
& \leq \mathbb{P}\left[T_{\left\lfloor 3 n M_{n}\right\rfloor} \leq 2 n M_{n}\right] \\
& \leq e^{-\operatorname{cst}^{\prime} \times n M_{n}} .
\end{aligned}
$$

The Borel-Cantelli Lemma thus gives: a.s.e., $\mathbf{d}\left(\mathfrak{S}_{n}^{M_{n}}\right)<\left\lfloor 3 n M_{n}\right\rfloor$.

It results from Lemma 3.7 applied to the estimate of Step 2 that a.s.e., $\mathfrak{d}_{t} \leq b t^{3}$. 
Step 4: Final bounds. Applying Lemma 3.6 to the polynomial estimates we now have yields the following: a.s.e., $\mathfrak{h}_{t} \leq \operatorname{cst}^{\prime \prime} \cdot t \ln t$. Applying Lemma 3.7 to this estimate gives the almost quadratic bound on the width.

Remark 3.8. The same arguments can be adapted to prove Theoremn 3.4 for any sufficiently horizontal initial cluster. More exactly, it is enough to assume that the initial cluster satisfies the geometric assumption given at the beginning of the section with $a<1$. In this case, the constant $c_{0}$ depends on $a$, the quantity $\mathfrak{h}_{t}$ stands for the maximal distance from a point of $\mathfrak{R}_{t}$ to $C$ and $\mathfrak{d}_{t}$ designates the diameter of $\mathfrak{R}_{t}$.

\section{Bounds on the height and width of the cluster}

Let us consider the discrete-time dynamics starting from $(0,0)$. In this section, let $\mathfrak{h}_{n}:=\mathbf{h}\left(C_{n}\right)$ and $\mathfrak{d}_{n}:=|\mathbf{d}|\left(C_{n}\right)$. Following Kesten (1987), we obtain the following bounds:

Theorem 4.1. For some constant $c_{1}$, almost surely, eventually,

$$
\begin{gathered}
\sqrt{2 n} \leq \mathfrak{h}_{n} \leq c_{1} n^{2 / 3} \\
\text { and } \\
c_{1}^{-1} n^{1 / 3} \leq \mathfrak{d}_{n} \leq c_{1} \sqrt{n} .
\end{gathered}
$$

Remark 4.2. For DLA, Kesten has proved that the radius of the cluster is almost surely eventually lower than $c_{1} n^{2 / 3}$.

Proof: Before applying Kesten's argument, we need some lower bound on the activity of the cluster. This is natural since a high activity of the cluster guarantees, for all $P \in \mathbb{Z}^{2}$, a low probability that this site will be the next to be added to the cluster (lower than $1 / \operatorname{act}($ cluster)). This allows us to control the probability that a path of length $l$ is added between times $n_{0}$ and $n_{1}$ and thus the probability that the height (or the width) of the cluster is increased by $l$ between $n_{0}$ and $n_{1}$.

Notice that the lower bounds are consequences of the upper bounds and the fact that $C_{n}$ contains $n+1$ particles.

Definition 4.3. An animal is a non-empty finite set that can be obtained by a DDLA starting from $(0,0)$.

Lemma 4.4. There is a constant $c$ such that

$$
\forall F \Subset \mathbb{Z}_{+}^{2}, F \text { is an animal } \Longrightarrow \operatorname{act}(F) \geq c \max (|\mathbf{d}|(F), \sqrt{\mathbf{h}(F)}) .
$$

Proof: First of all, we notice that

$$
\operatorname{act}(F)=\sum_{P \in L_{\mathbf{h}(F)}} 2 \mathbb{P}\left[\exists k \in \mathbb{Z}_{+}, P+W_{k} \in F\right],
$$

where $\left(W_{k}\right)$ is a downward directed symmetric random walk. This is a consequence of the equivalence between the two constructions of DDLA in discrete time.

We will prove that there exists $c \in \mathbb{R}_{+}^{\star}$ such that for every animal $F$ and every $Q \in L_{\mathbf{h}(F)}$,

$$
\mathbf{d}(Q) \in[-\sqrt{\mathbf{h}(F)}, 0] \Longrightarrow \mathbb{P}\left[\exists k \in \mathbb{Z}_{+}, Q+W_{k} \in F\right]>c .
$$


Together with the first formula of the proof, this will imply that

$$
\operatorname{act}(F) \geq c \sqrt{\mathbf{h}(F)} \text {. }
$$

Let $F$ be an animal and $P \in F$ be such that $\mathbf{h}(P)=\mathbf{h}(F)$. By symmetry, we can assume that $\mathbf{d}(P) \geq 0$. Since $F$ is an animal, for all $Q \in L_{\mathbf{h}(F)}$ such that $\mathbf{d}(Q)<\mathbf{d}(P)$, we have the following inequality:

$$
\mathbb{P}\left[\exists k \in \mathbb{Z}_{+}, Q+W_{k} \in F\right] \geq \mathbb{P}\left[\mathbf{d}\left(Q+W_{\mathbf{h}(F)}\right)>0\right] .
$$

Besides, if $\mathbf{d}(Q)>-\sqrt{\mathbf{h}(F)}$, then

$$
\mathbb{P}\left[\mathbf{d}\left(Q+W_{\mathbf{h}(F)}\right)>0\right] \geq \mathbb{P}\left[\tilde{W}_{\mathbf{h}(F)}>\sqrt{\mathbf{h}(F)}\right],
$$

where $\left(\tilde{W}_{k}\right)_{k}$ is the symmetric 1-dimensional random walk. The quantity in the right-hand side of this inequality is bounded from below by the Central Limit Theorem, implying half of the desired inequality.

Let $P \in F$ be such that $|\mathbf{d}(P)|=|\mathbf{d}|(F)$. Since $F$ is an animal, there exists $A \subset F$ such that $A$ is an animal, $\mathbf{h}(P)=\mathbf{h}(A)$ and $P \in A$. It follows from $(\star)$ that

$$
F \subset F^{\prime} \Longrightarrow \operatorname{act}(F) \leq \operatorname{act}\left(F^{\prime}\right) \text {. }
$$

Thus, we just need to prove the result for $A$.

By symmetry, we can assume that $\mathbf{d}(P)>0$. If $Q \in L_{\mathbf{h}(A)}$ and $\mathbf{d}(Q) \in[0, \mathbf{d}(P)]$,

$$
\mathbb{P}\left[\exists k, Q+W_{k} \in A\right] \geq \mathbb{P}\left[\mathbf{d}\left(Q+W_{\mathbf{h}(A)}\right)>0\right] \geq \frac{1}{2} .
$$

This ends the proof of the lemma.

Let $(\mathbf{f}, \alpha)$ be $\left(n \mapsto \mathbf{h}\left(A_{n}\right), \frac{1}{2}\right)$ or $\left(n \mapsto|\mathbf{d}|\left(A_{n}\right), 1\right)$. We will prove that there exists almost surely $k_{0}$ such that

$$
\forall k>k_{0}, \forall l, 2^{k} \leq l \leq 2^{k+1} \Longrightarrow \mathbf{f}\left(2^{k+1}\right)-\mathbf{f}(l) \leq \frac{2^{k+3}}{c \mathbf{f}(l)^{\alpha}}+2^{k / 2} .
$$

We then conclude using the following lemma.

Lemma 4.5 (discrete version of Gronwall's Lemma). Let $\alpha \in(0,1], c \in \mathbb{R}_{+}^{\star}$ and $\left(a_{n}\right)_{n \in \mathbb{Z}_{+}} \in \mathbb{Z}_{+}^{\mathbb{Z}_{+}}$. Assume that $\forall n, a_{n+1}-a_{n} \in[0,1]$ and that there exists $k_{0}$ such that

$$
\forall k>k_{0}, \forall l, 2^{k} \leq l \leq 2^{k+1} \Longrightarrow \frac{a_{2^{k+1}}-a_{l}}{2^{k}} \leq \frac{8}{c \cdot a_{l}^{\alpha}}+2^{-k / 2} .
$$

Then, there exists some $c_{1}$ depending only on $(\alpha, c)$ such that, eventually,

$$
a_{n} \leq c_{1} n^{1 /(\alpha+1)} .
$$

Its proof is postponed to the end of the section.

Let $(k, l)$ be such that $2^{k} \leq l \leq 2^{k+1}$ and let us set $m:=\left\lfloor\frac{2^{k+3}}{c \cdot \mathbf{f}(l)^{\alpha}}+2^{k / 2}\right\rfloor$. We are looking for an upper bound on $\mathbb{P}\left[\mathbf{f}\left(2^{k+1}\right)-\mathbf{f}(l)>m\right]$ in order to apply the Borel-Cantelli Lemma.

Definition 4.6. The path $\left(P_{1}, \ldots, P_{n}\right) \in\left(\mathbb{Z}_{+}^{2}\right)^{n}$ is said to be filled in order if

- it is an upward directed path: $\forall i, P_{i+1}-P_{i} \in\{(0,1),(1,0)\}$;

- all the $P_{i}$ belong to the considered cluster;

- if $i<j, P_{i}$ is added to the cluster before $P_{j}$. 
Assume that $\mathbf{f}\left(2^{k+1}\right)-\mathbf{f}(l)>m$. Let $P$ be such that

$$
\mathbf{f}(P)=\max _{Q \in C_{2^{k+1}}} \mathbf{f}(Q) .
$$

By construction of DDLA, there exists a path filled in order linking 0 to $P$. Taking its $r$ last steps for a suitable value of $r$, we obtain a path $\mathbf{P}=\left(P_{1}, \ldots, P_{r}\right)$ that is filled in order (relative to $C_{2^{k+1}}$ ) and such that $P_{r}=P$ and $\mathbf{f}\left(P_{r}\right)-\mathbf{f}\left(P_{1}\right)=m$. In particular, there exists a path of length $m-\left(P_{1}, \ldots, P_{m}\right)-$ filled in order such that its sites are added to the cluster between times $l$ and $2^{k+1}$.

The number of upward directed paths of length $m$ starting in $L_{\leq l}$ is

$$
\frac{(l+1)(l+2)}{2} \cdot 2^{m}
$$

We now need to control, for such a path $\mathbf{P}=\left(P_{1}, \ldots, P_{m}\right)$, the probability that it is filled in order between times $l$ and $2^{k+1}$. More precisely, we extend $\mathbf{P}$ to an infinite upward directed path and look for an upper bound on the probability that its first $m$ sites are successfully added between times $l$ and $2^{k+1}$. For $n \in\left[l+1,2^{k+1}\right]$, assume that $i=\min \left\{j \in \mathbb{N}: P_{j} \notin C_{n-1}\right\}$. Let $I_{n}$ be the event that $P_{i}$ is the site added at time $n$. The probability we want to control is lower than $\mathbb{P}\left[\sum_{n=l+1}^{2^{k+1}} I_{n} \geq m\right]$.

We know, by Lemma 4.4, that $\mathbb{P}\left[I_{n} \mid C_{n-1}\right] \leq \frac{1}{c \cdot \mathbf{f}(n-1)^{\alpha}}$. By monotonicity of $\mathbf{f}$, this implies that, almost surely,

$$
\sum_{n=l+1}^{2^{k+1}} \mathbb{P}\left[I_{n} \mid C_{n-1}\right] \leq \frac{2^{k}}{c \cdot \mathbf{f}(l)^{\alpha}} .
$$

We now use the following exponential bound:

Theorem 4.7 (Theorem 4.b in Freedman (1973)). Let $\left(\mathcal{F}_{n}\right)$ be a filtration. Let $\tau$ be an $\left(\mathcal{F}_{n}\right)$-stopping time. Let $\left(X_{n}\right)$ be a sequence of random variables such that

$$
\text { for every } n, X_{n} \in[0,1] \text { and } X_{n} \text { is } \mathcal{F}_{n} \text {-measurable. }
$$

Let $M_{n}:=\mathbb{E}\left[X_{n} \mid \mathcal{F}_{n-1}\right]$. Let $(a, b)$ be such that $0<b \leq a$. Then,

$$
\mathbb{P}\left[\sum_{n=1}^{\tau} X_{n} \geq a \text { and } \sum_{n=1}^{\tau} M_{n} \leq b\right] \leq\left(\frac{b}{a}\right)^{a} e^{a-b}
$$

Applying this to $I_{n}$ with $\mathcal{F}_{n}:=\sigma\left(C_{0}, \ldots, C_{n}\right), a:=m, b:=\frac{2^{k}}{c \cdot \mathbf{f}(l)^{\alpha}} \leq \frac{m}{8}$ and a constant stopping time, we obtain that the probability that there are at least $m$ successful fillings through $\mathbf{P}$ between times $l$ and $2^{k+1}$ is lower than $\left(\frac{e}{8}\right)^{m}$.

Thus,

$$
\begin{aligned}
\mathbb{P}\left[\mathbf{f}\left(2^{k+1}\right)-\mathbf{f}(l)>m\right] & \leq \frac{(l+1)(l+2)}{2} \cdot 2^{m} \cdot\left(\frac{e}{8}\right)^{m} \\
& \leq\left(2^{k+1}+2\right)^{2} \cdot\left(\frac{e}{4}\right)^{2^{k / 2}}
\end{aligned}
$$

Since $\sum_{k \geq 1} \sum_{l=2^{k}+1}^{2^{k+1}}\left(2^{k+1}+2\right)^{2} \cdot\left(\frac{e}{4}\right)^{2^{k / 2}}<\infty$, by the Borel-Cantelli Lemma and Lemma 4.5 , the proposition is established. 
Proof of Lemma 4.5: Take $d_{0}$ such that $\left(2^{1 /(\alpha+1)}-1\right) \cdot d_{0}>\frac{8}{c d_{0}^{\alpha}}+1$ and take $c_{1}>2^{1+1 /(\alpha+1)} d_{0}$. For $k>k_{0}$,

$$
\begin{aligned}
a_{2^{k}} \geq d_{0} \cdot 2^{k /(\alpha+1)} & \Longrightarrow a_{2^{k+1}}-a_{2^{k}} \leq \frac{8}{c} \cdot \frac{2^{k}}{d_{0}^{\alpha} \cdot 2^{k \alpha /(\alpha+1)}}+2^{k / 2} \\
& \Longrightarrow a_{2^{k+1}}-a_{2^{k}} \leq\left(\frac{8}{c d_{0}^{\alpha}}+1\right) \cdot 2^{k /(\alpha+1)} \\
& \Longrightarrow a_{2^{k+1}}-a_{2^{k}} \leq d_{0} \cdot 2^{(k+1) /(\alpha+1)}-d_{0} \cdot 2^{k /(\alpha+1)},
\end{aligned}
$$

where the last line results from the choice of $d_{0}$. Thus, there exists $k_{1}>k_{0}$ such that $a_{2^{k_{1}}} \leq 2 d_{0} \cdot 2^{k_{1} /(\alpha+1)}$.

If $\forall k \geq k_{1}, a_{2^{k}}>d_{0} \cdot 2^{k /(\alpha+1)}$, then the implication we have just proved shows that

$$
\forall k>k_{1}, a_{2^{k}}-a_{2^{k_{1}}} \leq d_{0} \cdot 2^{k /(\alpha+1)}-d_{0} \cdot 2^{k_{1} /(\alpha+1)},
$$

which implies that $\forall k \geq k_{1}, a_{2^{k}} \leq 2 d_{0} \cdot 2^{k /(\alpha+1)}$. Since $\left(a_{n}\right)_{n \in \mathbb{Z}_{+}}$is a non-decreasing sequence, we obtain

$$
\forall m>2^{k_{1}}, a_{m} \leq 2^{1+1 /(\alpha+1)} \cdot d_{0} \cdot m^{1 /(\alpha+1)} .
$$

Thus, we can assume that $k_{1}$ is such that $a_{2^{k_{1}}} \leq d_{0} \cdot 2^{k_{1} /(\alpha+1)}$. Assume that there exists $k_{2}>k_{1}$ such that $a_{2^{k_{2}}}>d_{0} \cdot 2^{k_{2} /(\alpha+1)}$. Take a minimal such $k_{2}$. By minimality, there exists some minimal $l$ between $2^{k_{2}-1}+1$ and $2^{k_{2}}$ such that $a_{l-1} \leq d_{0} \cdot(l-1)^{1 /(\alpha+1)}$ and $a_{l}>d_{0} \cdot l^{1 /(\alpha+1)}$. Thus,

$$
a_{2^{k_{2}}}-a_{l} \leq \frac{8}{c} \cdot \frac{2^{k_{2}-1}}{d_{0}^{\alpha} \cdot l^{\alpha /(\alpha+1)}}+2^{\left(k_{2}-1\right) / 2}
$$

and, since $a_{l} \leq a_{l-1}+1$,

$$
\begin{aligned}
a_{2^{k_{2}}} & \leq d_{0} \cdot(l-1)^{1 /(\alpha+1)}+1+\frac{8}{c} \cdot \frac{2^{k_{2}-1}}{d_{0}^{\alpha} \cdot l^{\alpha /(\alpha+1)}}+2^{\left(k_{2}-1\right) / 2} \\
& \leq 2 d_{0} \cdot 2^{k_{2} /(\alpha+1)}+1 .
\end{aligned}
$$

In fact, we have proved that, for $k \geq k_{1}$,

$$
\begin{aligned}
a_{2^{k}} \leq d_{0} \cdot 2^{k /(\alpha+1)} & \Longrightarrow a_{2^{k+1}} \leq 2 d_{0} \cdot 2^{(k+1) /(\alpha+1)}+1 \\
& \text { and } \\
a_{2^{k}}>d_{0} \cdot 2^{k /(\alpha+1)} & \Longrightarrow a_{2^{k}} \leq 2 d_{0} \cdot 2^{k /(\alpha+1)}+1 \\
& \Longrightarrow a_{2^{k+1}} \leq 2 d_{0} \cdot 2^{(k+1) /(\alpha+1)}+1 .
\end{aligned}
$$

This implies the proposition.

We can deduce from this a version of Theorem 4.1 for the continuous-time model. Of course, we set $\mathfrak{h}_{t}:=\mathbf{h}\left(C_{t}\right)$ and $\mathfrak{d}_{t}:=|\mathbf{d}|\left(C_{t}\right)$.

Proposition 4.8. For some constant $d_{1}$, almost surely, for every positive $\varepsilon$, eventually,

$$
\begin{gathered}
(2-\varepsilon) t \leq \mathfrak{h}_{t} \leq d_{1} t \\
\text { and } \\
\frac{\sqrt{t}}{d_{1}} \leq \mathfrak{d}_{t} \leq d_{1} t .
\end{gathered}
$$

Proof: The quantities $\mathfrak{h}_{t}$ and $\mathfrak{d}_{t}$ grow at most linearly because continuous-time DDLA is stochastically dominated by First-Passage Percolation.

If the lower extremity of an edge is a highest point of the cluster, then the activity of this edge is 1 . Consequently, if $T_{k}$ is the first time when the cluster is of height $k$, then $\left(T_{k+1}-T_{k}\right)_{k \in \mathbb{Z}_{+}}$is stochastically dominated by independent exponential 
random variables of parameter 2 (there exist at least 2 edges of lower extremity being a highest point of the cluster). This entails the at least linear growth of the height.

It results from this, the fact that discrete- and continuous-time DDLA define the same process and Theorem 4.1 that the number $N(t)$ of particles in the cluster at time $t$ satisfies, for some deterministic constant $a$,

$$
N(t) \geq a t^{3 / 2}
$$

almost surely eventually ${ }^{12}$. This implies that, a.s.e. $\mathfrak{d}_{t} \geq \frac{N(t)^{1 / 3}}{c_{1}} \geq \frac{a^{1 / 3}}{c_{1}} \sqrt{t}$.

\section{The infinite cluster}

Notation. In this section, we set

$$
S_{n}:=\left\{P \in \mathbb{Z}^{2}:\|P\|_{1}=n\right\} \text { and } B_{n}:=\left\{P \in \mathbb{Z}^{2}:\|P\|_{1} \leq n\right\} .
$$

We call elementary loop

$$
L:=\left\{P \in \mathbb{Z}^{2}:\|P\|_{\infty}=1\right\} .
$$

We start this section with a formal definition of (undirected) DLA.

Recall that if $F \Subset \mathbb{Z}^{2}$, the harmonic measure of $F$ is the unique probability measure $\mu_{F}$ such that the following holds:

Take any sequence $\left(\nu_{n}\right)$ of probability measures on $\mathbb{Z}^{2}$ satisfying

$$
\forall G \Subset \mathbb{Z}^{2}, \exists n_{G}, \forall n \geq n_{G}, \nu_{n}(G)=0 .
$$

Take $W_{n}$ the symmetric (non-directed) nearest-neighbor random walk in $\mathbb{Z}^{2}$, starting at 0 . Choose independently a starting point $P$ according to $\nu_{n}$. If $G$ is a non-empty subset of $Z^{2}$, let

$$
\tau(G)=\min \left\{k: W_{k} \in G\right\},
$$

which is finite almost surely. Then,

$$
\forall Q \in F, \mathbb{P}_{n}\left[P+W_{\tau(-P+F)}=Q\right] \underset{n \rightarrow \infty}{\longrightarrow} \mu_{F}(\{Q\}) .
$$

In words, $\mu_{F}$ measures the probability that a site in $F$ is the first site of $F$ to be touched by a walk launched from very far. For more information on the harmonic measure, see Spitzer (1976).

There are several equivalent ${ }^{13}$ definitions of DLA. The setting that will be convenient in this section is the following. The first cluster is $C_{0}:=\{(0,0)\} \subset B_{0}$. Assume that the first $n$ clusters have been built and are subsets of $B_{n}$. Independently of all the choices made so far, choose a point $P$ in $S_{n+2}$ according to $\mu_{S_{n+2}}$. Throw a symmetric random walk $\left(P+W_{k}\right)_{k \in \mathbb{Z}_{+}}$starting at $P$ and set

$$
C_{n+1}:=\left\{P+W_{\tau\left(-P+C_{n}\right)-1}\right\} \cup C_{n} \subset B_{n+1} .
$$

This process is called Diffusion-Limited Aggregation. ${ }^{14}$

\footnotetext{
12 because $N(t)$ goes to infinity when $t$ tends to infinity.

${ }^{13}$ The equivalences between the following definition and the natural definitions you may think of boil down to the definition of harmonic measure and strong Markov Property for random walks.

${ }^{14}$ The process consisting in adding a site with probability proportional to its harmonic measure relative to $\left\{P \notin C_{n}: \exists Q \in C_{n},\|P-Q\|_{1}=1\right\}$ is very similar to this process, but not equal to it in distribution.
} 
The following fact about $C_{\infty}:=\bigcup_{n} C_{n}$ is well-known.

Proposition 5.1. There is some $\varepsilon>0$ such that for all $P \in \mathbb{Z}^{2}, \mathbb{P}\left[P \notin C_{\infty}\right] \geq \varepsilon$.

Proof: Let $P \in \mathbb{Z}^{2}$. We consider our evolution temporally: we launch the first particle, look at it step after step until it sticks, before launching the second particle... A step is said to be critical if the current particle is at distance 1 from $P$ and $P$ is at distance 1 from the current cluster.

We wait for a critical step (we may wait forever). Conditionally on the fact that such a step exists, with probability $4^{-7}$, the particle tries — immediately after the first critical step - to visit all the points of $P+L$, say clockwise. ${ }^{15}$ Since the step is critical and $L$ has cardinality 8 , the particle must stick to some particle of the

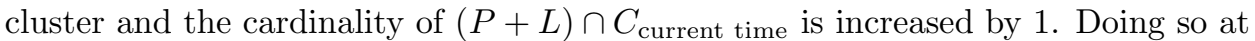
the first 8 critical steps that occur ${ }^{16}$ prevents $P$ from being added to the cluster. The fact thus holds for $\varepsilon:=4^{-7 \times 8}$.

Such a proof cannot work for the directed version of DLA. Indeed, take a site $P$ with a neighbor belonging to the cluster. Even assuming that there are enough particles coming in the neighborhood of $P$, one cannot always surround $P$ by modifying a finite number of steps: for example, $(2,0)$ will never be added to the cluster before $(1,0)$ if one considers a DDLA launched from $(0,0)$. The screening effect of the particles above it can be very strong, but will never reduce its activity to 0 .

However, we can prove the following proposition.

Proposition 5.2. Consider a DDLA starting from $\{(0,0)\}$. With positive probability, the site $(1,0)$ is never added to the cluster.

Proof: With positive probability, the first vertex to be added is $(0,1)$. Denote by $X_{t}$ the maximal first coordinate of an element of $\mathbb{Z}_{+} \times\{1\}$ that belongs to the cluster at time $t$. At time $t$, the activity of $(1,0)$ is at most $2^{1-X_{t}}$ times the activity of $\left(X_{t}+1,1\right)$. (To see this inequality, map a directed random walk $W$ launched at $(1,0)$ that takes its first $X_{t}$ steps to the left to the random walk launched at $\left(X_{t}+1,1\right)$ that merges with $W$ as soon as $W$ enters $\mathbb{Z}_{+} \times\{1\}$.) Thus, conditionally on the fact that $(n, 1)$ is added to the cluster before $(1,0)$, the probability that $(1,0)$ is added to the cluster before $(n+1,1)$ is at most $2^{-n}$. Since $\prod_{n \geq 1}\left(1-2^{-n}\right)$ is positive, Proposition 5.2 is established.

Corollary 5.3. Consider a DDLA starting from $\{(0,0)\}$. Almost surely, for every $n \in \mathbb{Z}_{+}$, only finitely many points of $\left(\mathbb{Z}_{+} \times\{n\}\right) \cup\left(\{n\} \times \mathbb{Z}_{+}\right)$are added to the cluster.

Notation. Recall that for $b \in \mathbb{R}_{+}^{*}$, we set $\mathcal{C}_{b}:=r_{-\pi / 4}\left(\left\{(x, y) \in \mathbb{R}^{2}:|y| \geq b|x|\right\}\right)$.

Proposition 5.4. Consider a DDLA starting from $C \neq \varnothing$. Let

$$
C_{\infty}:=\bigcup_{t \geq 0} C_{t} .
$$

Then, almost surely, for all $P \in \mathbb{Z}^{2}$, for all $b>0, C_{\infty} \cap\left(P+\mathcal{C}_{b}\right)$ is infinite.

\footnotetext{
${ }^{15} \mathrm{By}$ this, we mean that the following 7 steps that the particle would take if it was not hindered by the cluster are the ones making it visit $P+L$ clockwise.

16 which means at every critical step if there are less than 8 of them

By "the first 8 critical steps", we mean the first critical step (which occurs for the $k_{1}^{\text {th }}$ particle), the first critical step of a particle different from the $k_{1}^{\text {th }}$ one, and so on up to 8 .
} 
Proof: Notice that it is enough to prove the fact with "non-empty" instead of "infinite".

There is an increasing path $\mathbf{P}=\left(P_{1}, \ldots, P_{n}\right)$ going from a point of $C$ to a point in $P+\mathcal{C}_{b}$. The conic structure and the law of large numbers guarantee that the activity of $P_{n}$ is bounded away from 0 (say larger than $c>0$ ) as long as $P+\mathcal{C}_{b}=\varnothing$ (which we now assume).

Thus, if $k(t):=\max \left\{i: P_{i} \in C_{t}\right\}$ and if $k(t)<n$, then $P_{k(t)+1}$ will be added at rate at least $2^{n-k(t)} c>0$. Indeed, a walk can reach $P_{n}$ from $P_{k+1}$ by using $\mathbf{P}$; then, from $P_{n}$, it escapes with probability $c$. Consequently, $k(t)$ will almost surely take a finite time to increase its value, as long $k(t)<n$. Thus $k(\infty)=n$, and Proposition 5.4 is established.

Let us conclude with a couple of questions.

Question 5.5. For which values of $b$ does it hold that the infinite DDLA cluster is almost surely a subset of $\mathcal{C}_{b}$ up to finitely many points?

Question 5.6. What is the distribution of the number of ends of the infinite DDLA cluster?

\section{Acknowledgements}

I thank Vincent Beffara for proposing this topic of research to me, as well as for his advice and availability. I am indebted to Vincent Beffara and Jessica Todd for allowing me to use in this paper some of their pictures.

\section{References}

G. Amir. One-dimensional long-range diffusion-limited aggregation III: the limit aggregate (2017+). To appear in Ann. de l'Inst. H. Poincaré.

G. Amir, O. Angel and G. Kozma. One-dimensional long-range diffusion limited aggregation II: the transient case. ArXiv Mathematics e-prints (2013). arXiv: 1306.4654 .

Gideon Amir, Omer Angel, I. Benjamini and Gady Kozma. One-dimensional longrange diffusion-limited aggregation I. Ann. Probab. 44 (5), 3546-3579 (2016). MR3551204.

I. Benjamini and A. Yadin. Diffusion limited aggregation on a cylinder. Comm. Math. Phys. 279 (1), 187-223 (2008). MR2377633.

N. Berger, J. J. Kagan and E. B. Procaccia. Stretched IDLA. ALEA Lat. Am. J. Probab. Math. Stat. 11 (1), 471-481 (2014). MR3274641.

R. Bradley and P. Strenski. Directed diffusion-limited aggregation on the bethe lattice: Exact results. Phys. Rev. B 30,6788-6790 (1984). DOI: 10.1103/PhysRevB.30.6788.

R. Bradley and P. Strenski. Directed aggregation on the Bethe lattice: Scaling, mappings, and universality. Phys. Rev. B 31, 4319-4328 (1985). DOI: 10.1103/PhysRevB.31.4319.

R. Brady and R. Ball. Fractal growth of copper electrodeposits. Nature 309, 225-229 (1984). DOI: 10.1038/309225a0. 
L. Carleson and N. Makarov. Laplacian path models. J. Anal. Math. 87, 103-150 (2002). MR1945279.

P. Diaconis and W. Fulton. A growth model, a game, an algebra, Lagrange inversion, and characteristic classes. Rend. Sem. Mat. Univ. Politec. Torino 49 (1), 95-119 (1993) (1991). MR1218674.

D. M. Eberz-Wagner. Discrete growth models. ProQuest LLC, Ann Arbor, MI (1999). ISBN 978-0599-38096-7. Thesis (Ph.D.)-University of Washington. MR2699374.

D. Freedman. Another note on the Borel-Cantelli lemma and the strong law, with the Poisson approximation as a by-product. Ann. Probability 1, 910-925 (1973). MR0370711.

F. Johansson Viklund, A. Sola and A. Turner. Scaling limits of anisotropic HastingsLevitov clusters. Ann. Inst. Henri Poincaré Probab. Stat. 48 (1), 235-257 (2012). MR2919205.

H. Kesten. Aspects of first passage percolation. In École d'été de probabilités de Saint-Flour, XIV-1984, volume 1180 of Lecture Notes in Math., pages 125-264. Springer, Berlin (1986). MR876084.

H. Kesten. How long are the arms in DLA? J. Phys. A 20 (1), L29-L33 (1987). MR873177.

G. F. Lawler, M. Bramson and D. Griffeath. Internal diffusion limited aggregation. Ann. Probab. 20 (4), 2117-2140 (1992). MR1188055.

S. Majumdar. Traveling front solutions to directed diffusion-limited aggregation, digital search trees, and the Lempel-Ziv data compression algorithm. Physical Review E 68 (2), 026103 (2003). DOI: 10.1103/PhysRevE.68.026103.

T. Seppäläinen. Strong law of large numbers for the interface in ballistic deposition. Ann. Inst. H. Poincaré Probab. Statist. 36 (6), 691-736 (2000). MR1797390.

B. Shraiman and D. Bensimon. Singularities in nonlocal interface dynamics. Phys. Rev. A (3) 30 (5), 2840-2842 (1984). MR763133.

R. T. Smythe and John C. Wierman. First-passage percolation on the square lattice, volume 671 of Lecture Notes in Mathematics. Springer, Berlin (1978). ISBN 3540-08928-4. MR513421.

F. Spitzer. Principles of random walk. Springer-Verlag, New York-Heidelberg, second edition (1976). MR0388547.

T. Vicsek. Fractal growth phenomena. World Scientific Publishing Co., Inc., River Edge, NJ, second edition (1992). ISBN 981-02-0668-2; 981-02-0669-0. MR1182314.

T. Witten and L. Sander. Diffusion-Limited Aggregation, a Kinetic Critical Phenomenon. Phys. Rev. Lett. 47, 1400-1403 (1981). DOI: 10.1103/PhysRevLett.47.1400. 